\title{
Spatially Resolved STIS Spectroscopy of Betelgeuse's Outer Atmosphere
}

\author{
A. Lobel, J. Aufdenberg, A. K. Dupree, R. L. Kurucz, R. P. Stefanik, \& \\ G. Torres \\ Harvard-Smithsonian Center for Astrophysics, 60 Garden Street, \\ Cambridge 02138 MA, USA
}

\begin{abstract}
.
We present spatially resolved spectra observed with the Space Telescope Imaging Spectrograph on the Hubble Space Telescope of the upper chromosphere and dust envelope of Betelgeuse ( $\alpha$ Orionis, M2 Iab). In the fall of 2002 a set of five high-resolution near-UV spectra was obtained by scanning at intensity peak-up position and four off-limb target positions up to one arcsecond, using a small aperture (200 by 63 mas), to investigate the thermal conditions and flow dynamics in the outer atmosphere of this important nearby cool supergiant star.

Based on $\mathrm{Mg}$ II $h \& k, \mathrm{Fe}$ II $\lambda 2716, \mathrm{C}$ II $\lambda 2327$, and $\mathrm{Al}$ II ] $\lambda 2669$ emission lines we provide the first evidence for the presence of warm chromospheric plasma at least 1 arcsecond away from the star at $\sim 40$ $\mathrm{R}_{*}\left(1 \mathrm{R}_{*} \simeq 700 \mathrm{R}_{\odot}\right)$. The STIS spectra reveal that Betelgeuse's upper chromosphere extends far beyond the circumstellar $\mathrm{H} \alpha$ envelope of $\sim 5 \mathrm{R}_{*}$, determined from previous ground-based imaging (Hebden et al. 1987).

The flux in the broad and self-absorbed resonance lines of $\mathrm{Mg}$ II decreases by a factor of $\sim 700$ compared to the flux at chromospheric disk center. We observe strong asymmetry changes in the $\mathrm{Mg}$ II $h$ and Si I resonance line profiles when scanning off-limb, signaling the outward acceleration of gas outflow in the upper chromosphere.

From the radial intensity distributions of Fe I and Fe II emission lines we determine the radial non-LTE iron ionization balance. We compute that the local kinetic gas temperatures of the warm chromospheric gas component in the outer atmosphere exceed $2600 \mathrm{~K}$, when assuming local gas densities of the cool gas component we determine from radiative transfer models that fit the $9.7 \mu \mathrm{m}$ silicate dust emission feature. The spatially resolved STIS spectra directly demonstrate that warm chromospheric plasma co-exisists with cool gas in Betelgeuse's circumstellar dust envelope.
\end{abstract}

\section{STIS Observations}

STIS spectra of the red supergiant $\alpha$ Ori have been observed for GO 9369 in HST Cycle 11; A direct Test for Dust-driven Wind Physics. This program investigates the detailed acceleration mechanisms of wind outflow in the outer atmospheres (chromosphere and dust envelope) of cool stars. Using the exceptional capabilities of HST-STIS we observe the near-UV spectrum with $\lambda / \Delta \lambda \simeq 33,000$ between 

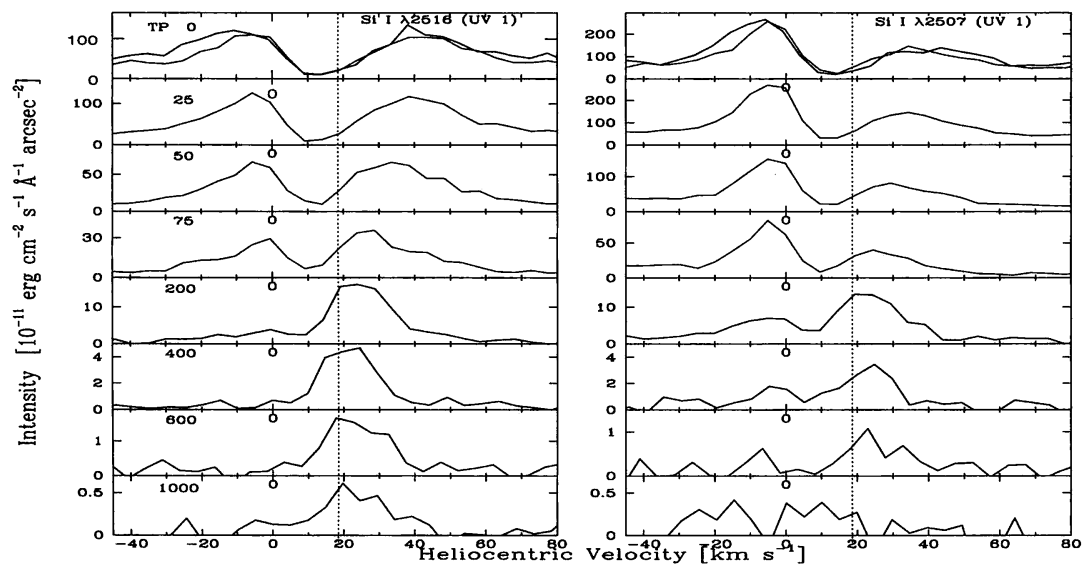

Figure 1. Spatially resolved STIS spectra of Si I $\lambda 2516$ and Si I $\lambda 2507$ resonance emission lines observed in Betelgeuse out to 1 arcsecond.

$2275 \AA$ and $3180 \AA$ with spatially resolved scans across the chromospheric disk at $0,200,400,600, \& 1000$ mas (Visit 1), at $0 \& 2000$ mas (Visit 2), and at $0 \& 3000$ mas (Visit 3). We presently discuss the spectra observed in fall 2002 of Visit 1. The spectra of Visits $2 \& 3$ of spring 2003 will be presented elsewhere. Exposure times range from $500 \mathrm{~s}$ at 200 mas to $7200 \mathrm{~s}$ at $1^{\prime \prime}$, yielding good $\mathrm{S} / \mathrm{N} \geq 20$. The spectra are calibrated with CALSTIS v2.12 using the most recently updated calibration reference files. Wavelength calibration accuracies are better than $\sim 1$ detector pixel or $1.3 \mathrm{~km} \mathrm{~s}^{-1}$.

\section{Si I $\lambda 2516$ line profile changes}

In previous work we modeled the detailed shape of the $\mathrm{Si}$ I $\lambda 2516$ resonance emission line (Lobel \& Dupree 2001). The line has previously been observed by scanning over the inner chromosphere at $0,25,50$, and 75 mas, using a slit size of $100 \times 30$ mas (Fig. 1, panel left). The figure at cfa-www.harvard.edu/cfa/ep/ pressrel/alobelimg.html shows the line profiles of Fe II $\lambda 2869$ for the respective slit positions compared to the near-UV continuum flux observed with HST-FOC. The double-peaked line profiles are observed across the inner chromosphere. The central (self-) absorption core results from scattering opacity in the chromosphere. The asymmetry of the emission component intensities probes the chromospheric flow dynamics in our line of sight. The spectra of GO 9369 are observed across the outer chromosphere using a slitsize of $200 \times 63$ mas (Fig. 1). The profiles beyond 200 mas appear red-shifted with a rather weak shortwavelength emission component. It signals substantial wind outflow opacity in the upper chromosphere, which accelerates rapidly beyond a radius of $\sim 8 R_{*}$.

\section{3. $\quad \mathrm{Mg}$ II $k \& h$ line profile changes}

Figure 2 shows the detailed profiles of the $\mathrm{Mg}$ II $h \& k$ lines observed up to 1000 mas. The emission line intensities decrease by a factor of $\sim 700$ from chromospheric disk center (TP 0 ) to $1^{\prime \prime}$. These optically thick chromospheric lines show remarkable changes of their detailed shapes when scanning off-limb. The full 


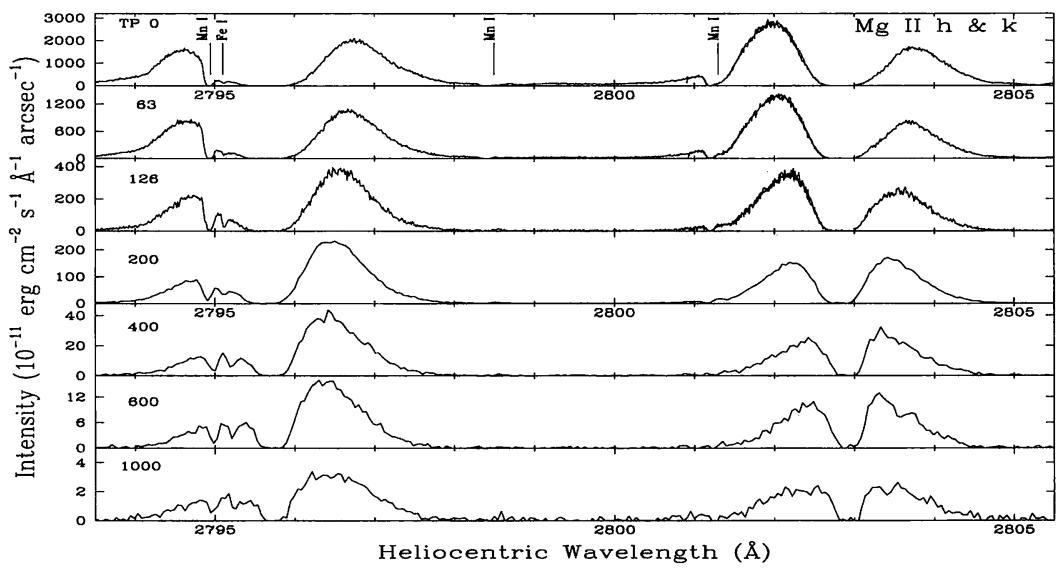

Figure 2. $\quad$ Spatially resolved STIS spectra of $\mathrm{Mg}$ II $h \& k$ emission lines observed in the chromosphere of Betelgeuse out to 1 arcsecond.

width across both emission components at half intensity maximum decreases by $\sim 20 \%$, while the broad and saturated central absorption core narrows by more than $50 \%$. Beyond 600 mas the central core assumes a constant width which results from absorption contributions by the local interstellar medium $\left(d_{*} \simeq 132 \mathrm{pc}\right)$. We observe a strong increase of the (relative) intensity of the long-wavelength emission component in both lines beyond 200 mas. It signals fast wind acceleration beyond this radius. Note that the short-wavelength emission components of the $k$ and $h$ lines are blended with chomospheric Mn I lines (decreasing the $k$ - and increasing the $h$-component), but that become much weaker in the outer chromosphere.

\section{Wind Acceleration in the Upper Chromosphere}

Figure 1 compares the profiles of the Si I $\lambda 2516$ and $\lambda 2507$ resonance lines (vertical dotted lines are drawn at stellar rest velocity). Both lines share a common upper energy level and their intensities are influenced by pumping through a fluoresced Fe II line. The self-absorption cores of the Si I lines are therefore observed far out, into the upper chromosphere. The shape of these unsaturated emission lines is strongly opacity sensitive to the local chromospheric velocity field. As for the $\mathrm{Mg}$ II lines, the outward decreasing intensity of the short-wavelength emission component signals fast acceleration of chromospheric outflow in the upper chromosphere. We also observe this decrease for the resonance line of Mg I $\lambda 2852$ (not shown). Our previous radiative transfer modeling work based on $\mathrm{Si}$ I revealed that $\alpha$ Ori's inner chromosphere oscillates non-radially, with simultaneous up- and downflows in Sept. 1998. Radiative transfer modeling to determine the detailed wind structure in the outer chromosphere is underway.

\section{Ion lines in the Upper Chromosphere}

We also observe ion lines of Fe II, Al II, and C II out to $1^{\prime \prime}$ in the upper chromosphere. Figure 3 shows (scaled) emission lines of Fe II $\lambda 2716$ (UV 62), Al II ] $\lambda 2669$ (UV 1), and C II $\lambda 2327$ (UV 1). The Fe I $\lambda 2823$ (UV 44) line is also shown for comparison (top panels). The lines at the inner chromosphere are observed 

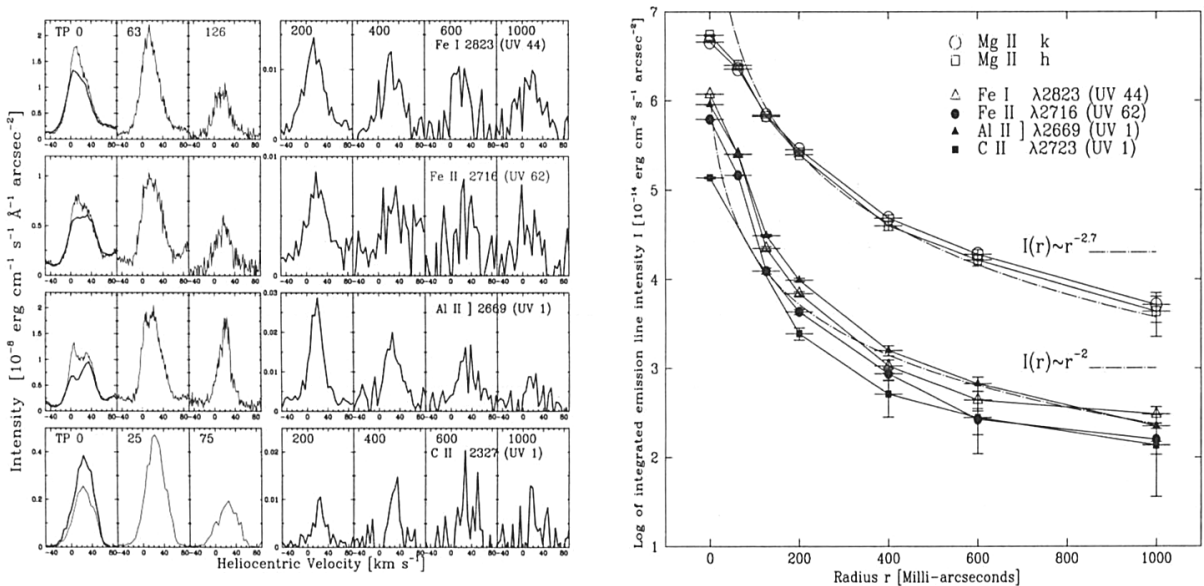

Figure 3. panel left: Spatially resolved chromospheric emission lines of $\mathrm{Fe} \mathrm{I}, \mathrm{Fe}$ II, $\mathrm{Al}$ II ], and $\mathrm{C}$ II. Figure 4. panel right: Radial chromospheric intensity distribution of these lines compared to $\mathrm{Mg}$ II (Fig. 2).

in April 1998 (thin drawn lines) with $\mathrm{R} \sim 114,000$ at TPs 0, 63, and 126 mas, while the lines of the outer chromosphere are observed with medium resolution in fall 2002 (boldly drawn lines). Both raster scans are however observed with the same slitsize of $200 \times 63$ mas so that the line intensity changes can be compared. For this purpose we select unblended lines without central self-absorption cores that become sufficiently optically thin in the outer chromosphere, and that are significantly observed against the local background noise level.

\section{Radial Intensity Distribution of Ion Lines}

We wavelength integrate the selected chromospheric emission lines and the $\mathrm{Mg}$ II lines beyond the line wings. Their radial intensity distributions $\mathrm{I}(\mathrm{r})$ are compared in Fig. 4. The intensity errorbars are derived from the STIS pipeline flux calibration errors, while the radius errorbars are derived from the projected slitwidth. We observe that the I(r) of optically thin emission from neutral and ion lines are very similar across the chromosphere. Neutral emission lines are generally observed farther out with larger $\mathrm{S} / \mathrm{N}$ compared to the ion lines, but their $\mathrm{I}(\mathrm{r})$ do not differ significantly within the errors. We find a best fit for $\mathrm{I}(\mathrm{r}) \simeq$ const $\times \mathrm{r}^{-2}$. The $\mathrm{I}(\mathrm{r})$ of the optically thick and self-absorbed $\mathrm{Mg}$ II lines differs significantly with $\mathrm{I}(\mathrm{r}) \simeq$ const $\times \mathrm{r}^{-2.7}$. The steeper intensity distribution signals important radiative transfer effects for the shapes of the stronger $\mathrm{Mg}$ II lines (see Sect. 3).

\section{Radial Non-LTE Iron Ionization Balance}

In the upper panel of Fig. 5 we compute the iron ionization fraction from the $\mathrm{I}(\mathrm{r})$ of the $\mathrm{Fe} \mathrm{I}$ and $\mathrm{Fe}$ II lines. The intersection of the curves (at dots) provides the excitation temperature corresponding to the observed line intensity ratios for spontaneous emission. We compute iron ionization fractions between $99.3 \%$ and $99.7 \%$ for kinetic gas temperatures between $2600 \mathrm{~K}$ and $5800 \mathrm{~K}$, using local gas densities $10^{-17} \leq \rho \leq 10^{-15} \mathrm{gr} \mathrm{cm}^{-3}$ (lower panel). This temperature range corresponds to partial NLTE iron ionization due to a diluted radiation field 

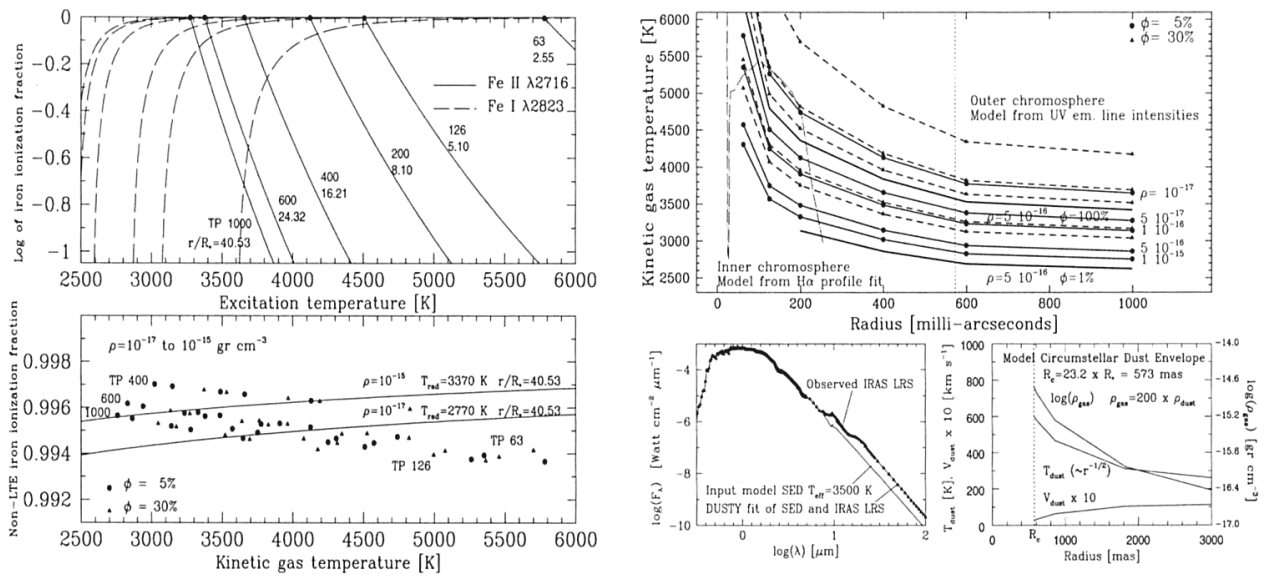

Figure 5. panels left: Non-LTE iron ionization fraction with kinetic gas temperature determined from I(r). Figure 6. panels right: Temperature structure of the chromosphere computed for different densities.

with $T_{\mathrm{r} \mathrm{d}} \simeq 3000 \mathrm{~K}$ (full drawn lines), typical for the outer chromosphere. The graphs are computed with volume filling factors $\phi$ for warm plasma of $5 \%$ (solid dots) and $30 \%$ (solid triangles). Hydrogen is almost neutral for these conditions in the upper chromosphere. In Fig. 6 we model the circumstellar dust envelope (CDE) with radiative transfer in spherical geometry using DUSTY. A best fit to the IRAS silicate dust emission feature at $9.7 \mu \mathrm{m}$ yields a dust condensation radius of $R_{c} \simeq 573$ mas, where $\rho_{\mathrm{g} \mathrm{s}} \sim 5 \times 10^{-16} \mathrm{gr} \mathrm{cm}^{-3}$ for the cool ambient gas, with temperatures below $T_{\text {dust }} \leq 600 \mathrm{~K}$ (lower panels). The upper panel shows the temperature structure for warm chromospheric plasma computed at this $\rho_{\mathrm{g}} \mathrm{s}$ with $\phi=1 \%$ and $100 \%$ (bold solid lines). The inner chromosphere is computed with radiative transfer fits to $\mathrm{H} \alpha$ (Lobel \& Dupree 2000). We find that the temperature of warm chromospheric plasma cannot decrease to below $2600 \mathrm{~K}$ in the CDE. Hence warm chromospheric plasma must co-exisit with cool gas of $T \leq 600 \mathrm{~K}$ beyond 600 mas.

\section{Conclusions}

Spatially resolved spectra of Betelgeuse's outer chromosphere signal that it accelerates outwards, based on asymmetries observed in $\mathrm{Mg}$ II $h \& k$, and other emission lines. They also reveal warm chromospheric plasma out to $40 \mathrm{R}_{*}$. The chromospheric gas must co-exist with cool gas of the circumstellar dust envelope.

Acknowledgments. This reseach is based on data obtained with the NASA/ ESA Hubble Space Telescope, collected at the STScI, operated by AURA Inc., under contract NAS5-26555. Financial support is provided by STScI grant HSTGO-09369.01 to the Smithsonian Astrophysical Observatory.

\section{References}

Lobel, A., \& Dupree, A.K. 2000, ApJ, 545, 454

Lobel, A., \& Dupree, A.K. 2001, ApJ, 558, 815

Hebden, J. C., Eckart, A., \& Hege, E. K. 1987, ApJ, 314, 690 